\title{
Special Session on Computational Fetal Monitoring
}

\author{
Joachim A Behar ${ }^{1}$, Zeev Weiner ${ }^{2}$ and Philip Warrick ${ }^{3}$ \\ ${ }^{1}$ Faculty of Biomedical Engineering, Technion, Israel Institute of Technology, Haifa, Israel \\ ${ }^{2}$ Department of Obstetrics and Gynecology, Rambam Medical Center, Haifa, Israel \\ ${ }^{3}$ PeriGen, Cranbury, NJ, and Westmount, QC, Canada
}

\begin{abstract}
Despite the improvements made in perinatal medicine during the 20th century, stillbirths still occur for 1 in 160 pregnancies in the US which represents a total of 26,000 fetal deaths each year. In addition, approximately 1 in 1000 fetuses experience oxygen deprivation during labor which is severe enough to cause brain injury. It is estimated that half of these cases of birth-related injury are preventable. Incorrect cardiotocography $(C T G)$ interpretation is leading the list of causes.

Intrapartum CTG is used routinely to measure maternal uterine pressure and fetal heart rate (FHR). Antepartum CTG monitoring is used to identify fetuses at risk of intrauterine hypoxia and acidaemia. As early as 28 weeks of gestation, analysis of the FHR trace is used as a nonstress test to assess the fetal well-being. In the perinatal period, timely, appropriate intervention can avoid fetal neurological damage or death. The CTG is visually assessed by a clinician or interpreted by computer analysis. In the context of labor monitoring, the CTG is used for continuous fetal monitoring. An abnormal heart rate will lead the clinician to perform a cesarean.

With the recent advances in machine learning and statistical signal analysis new algorithms for assessing fetal antepartum or intrapartum health status are being elaborated. These algorithms process signals recorded by CTG monitors or alternative monitoring techniques such as scalp electrocardiography or non-invasive fetal electrocardiography. This session discusses the history of fetal monitoring, its current challenges and the prospects opened by recent algorithmic development.
\end{abstract}

\section{Introduction}

In the US, stillbirths occur for 1 in 160 pregnancies and over half of all these are caused by obstetric conditions and placental abnormalities [1]. This represents a total of 26,000 fetal deaths each year in the US alone [2]. The prevalence of stillbirths may be up to 10 times higher in developing countries. Further, recent independent studies in the US and Portugal estimate that for 1 in 1000 births, oxygen deprivation during labor is severe enough to cause brain injury (hypoxic ischemic encephalopathy) [3], [4].
With the recent advances in machine learning and statistical signal analysis new algorithms for assessing fetal antenatal or labor health status are being elaborated. These algorithms use signals recorded by standard of care CTG monitoring and alternative monitoring techniques such as scalp electrocardiography or non-invasive fetal electrocardiography (NI-FECG).

This session aims to discuss the history of fetal monitoring, its current challenges and prospects opened by recent algorithmic developments to improve fetal monitoring. Specifically, we will focus on continuous fetal monitoring techniques and mainly focus on fetal heart rate analysis. We will distinguish between antepartum (the time period before childbirth) and intrapartum (from the onset of labor through to the delivery of the placenta) fetal monitoring.

\section{History of fetal heart rate monitoring}

In the 17th century, Marsac, a French physician is the first credited with having heard the fetal heart. In 1821, Lejumean Kergaradec, a French Nobleman described fetal heart tones and suggested its usage for auscultation. In 1906 the fetal electrocardiogram was observed for the first time in Germany by Cremer [5]. In 1917 the design of the "Fetoscope", a vaginal stethoscope, was published by David Hillis [6]. In 1958, Hon [7] analyzed the errors made by 15 obstetricians in estimating the fetal heart rate by auscultation. He demonstrated that significant errors are made particularly at the slowest and fastest fetal heart rate (FHR). His results highlighted the unreliability of humans in accurately computing the FHR. Hon emphasized that the use of electronic monitor during labor and delivery may permit a more valuable and accurate identification of fetal distress. The era of electronic fetal monitoring (EFM) had begun.

The work of Hon in the 1950s led him to develop the first EFM that could continuously measure the FHR and uterine activity. Observational studies conducted in the 1960s demonstrated a decrease in intrapartum stillbirth rates when EFM was performed. This led to its widespread acceptance in the clinical practice for both high-risk laboring and low-risk laboring women [8]. In 1968 Hewlett 
Packard and Hammacher produced the first clinically practical and commercially available monitor using phonocardiography and tocodynamometry for widespread purchase and use [9]. In the 1970s and 80s, randomized trials compared EFM to intermittent auscultation using a Pinard stethoscope or hand-held Doppler. EFM was not associated with better outcomes (e.g., APGAR or perinatal mortality), but resulted in an increase in the number of caesarean sections (CS). Despite these findings, EFM use became widespread [8] and ultimately ubiquitous by the 1990s.

Since the 1990s, more research has been performed to assess the usefulness of CTG in monitoring fetal health during labor. A recent Cochrane meta-analysis [10] of randomized and quasi-randomized controlled trials concluded that CTG during labour is associated with reduced rate of neonatal seizures but there are no clear differences in cerebral palsy, infant mortality or other measures of neonatal wellbeing. However, CTG during labour is associated with an important increase in CS rates. This study also pointed out that the impact of CTG on other long-term neurodevelopmental outcomes still needs to be evaluated. These trials led to the controversial situation of challenging the benefit of EFM versus periodic auscultation. Yet in current obstetrical practice, decision making continues to rely on EFM during labor.

In order to address the modern challenges associated with continuous fetal monitoring, efforts have been made in:

- Standardizing the interpretation of fetal heart rate traces such as the FIGO guidelines [11]. This also includes a better training of health professionals in interpreting these traces.

- Developing alternative monitoring techniques, with the key idea of providing more reliable or novel signals that may be used concomitantly or as a replacement to $\mathrm{CTG}$.

- Development of new computational fetal monitoring algorithms for the purpose of, better extracting the signal of interest (e.g. NI-FECG), identifying biomarkers that enable to assess the fetal status (e.g. distress, growth restriction, cardiac arrhythmia) and for providing decision making support.

\section{Alternative monitoring techniques}

In order to address the limitations of the CTG, alternative continuous fetal monitoring techniques have been developed, namely, fetal oximetry, the STAN scalp electrocardiography monitor (Neoventa Medical) and noninvasive fetal electrocardiography (NI-FECG) monitors.
However, these alternative techniques have not to date shown a significant improvement over the conventional CTG usage. With fetal oximetry, the study from Bloom et al. [12] has shown that knowledge of the fetal oxygen saturation is not associated with a reduction in the CS rate or with improvement in the condition of the newborn. Since then, there has not been much attempt to move this technology forward.

In scalp ECG analysis, it is hypothesized that an elevation of the ST segment and the ratio between the $\mathrm{T}$ wave and QRS amplitudes (T/QRS), may identify fetal anaerobic myocardial metabolism. The conclusion of a recent Cochrane review (2015) [13] was that strong evidence was lacking to demonstrate that ST waveform analysis had an effect on the primary outcome measures. However, fewer scalp samples during labor were made. Today, scalp ECG is in use in a number of hospitals around the world.

In NI-FECG, there has been technological challenges in robustly separating the fetal signal from the abdominal mixture because of the low signal to noise ratio of the fetal signal sensed on the abdomen and the presence of the isolating vernix caseosa layer during the third trimester of the pregnancy [14], [15]. However, recent advances in sensing technology and electrodes placement as well as advanced source separation algorithms have made this modality more and more attractive and many startups have obtained CE/FDA regulatory approval for their NI-FECG monitor [16].

\section{Computational fetal monitoring}

\section{Early work on antepartum computerized CTG}

Seminal work in the 1980 s in computational fetal monitoring for the antepartum period in Oxford [17] led to the development of the Dawes-Redman criteria for antepartum fetal wellbeing assessment which has been commercialized by Huntleigh Healthcare Ltd. It is currently used in the UK, Europe, the Middle and Far East. In particular, the short term variability (STV) and long term variability (LTV) measures elaborated in [18] have shown to be relevant indicators of fetal wellbeing.

\section{Intrapartum computerized CTG}

In the past years the Oxford group has been doing important work in computational antepartum monitoring using a data-driven approach. They hypothesized that computerized CTG can outperform clinical diagnosis of "fetal distress". Evaluation of the latest version of the OxSys (1.5) system on a large dataset of 22,790 CTG recordings from women in labor ( $\geq 36$ weeks GA) has shown good results [19]. It increased the sensitivity for compromise detection: $43.3 \%$ vs. $38.0 \%$ for severe ( $p=$ 
$0.3)$ and $36.1 \%$ vs. $31.0 \%$ for moderate $(p=0.06)$; and reduced the false-positive rate $(14.4 \%$ vs. $16.3 \%, \mathrm{p}<$ 0.001). The main computerized parameter in the computational system was the decelerative capacity (DC) of the phase rectified signal averaging (PRSA) [20].

Other interesting computerized CTG monitors include the Omniview-SisPorto (Speculum, Lisbon, Portugal) and PeriWatch Vigilance (PeriGen, Cary, North Carolina). The Omniview-SisPorto system implements a computerized version of the updated FIGO guidelines. In its latest version a combination of CTG analysis with ST event features is used to provide online alerts [21]. The PeriWatch Vigilance implements a 5-tier, color-coded graded classification of the CTG trace using automated estimates of the classical clinical features such as baseline, accelerations and decelerations [22]. Another interesting work looked at the dynamic relationship between intrapartum uterine pressure and fetal heart rate to distinguish between normal and hypoxic fetuses [23]. A recent review on intrapartum computerized monitoring is available in [24].

\section{Non-invasive fetal electrocardiography}

Advances in signal processing and machine learning have made FHR extraction from the NI-FECG more robust. With these advances new CE/FDA approved commercial monitors such as the Monica (Monica Healthcare Ltd), Meridian (MindChild Medical Inc) and Nemo (Veghel, the Netherlands) systems have become available. In computational NI-FECG, new algorithms to reconstruct the FECG morphology are being elaborated [25], [26] and evaluated against the measurements of ECG intervals as recorded from the scalp FECG [27], [28]. In addition, new source separation [29] and fetal heart beat detection algorithms [30] are being elaborated for this task. Finally, the NI-FECG is being evaluated for both antepartum and intrapartum monitoring and diagnosis of fetal pathologies such as fetal arrhythmias [31].

\section{Heart rate variability measures}

We pointed to the relevance of STV, LTV [18] and DC [20] heart rate variability (HRV) measures in computerized CTG analysis. Other HRV measures which have shown interesting usage include the scattering transform for intrapartum fetal monitoring $(n=45)$ [32] and entropy measures (approximate and sample entropy) for IUGR screening ( $n=59)$ [33]. A good summary of HRV measures used in the context of fetal HRV analysis for diagnosis and monitoring is available in Hoyer et al. [34].

\section{Conclusion}

The FHR conveys important information about the health of the fetus. Continuous monitoring of the FHR became widespread and ubiquitous in developed countries; however, it did not meet the expectations of the medical community and led to a dramatic increase in CS rates. Consequently, in recent years, there has been a significant controversy about its benefit. However, it is interesting to note that since its introduction in clinical practice the number of perinatal deaths has significantly decreased although the causal link is not clear. Alternative continuous monitoring methods have been created to tackle the limitations of CTG. This includes the short-lived fetal oximetry monitoring technique and the fetal scalp electrocardiography which is in use in many hospitals. NIFECG is another candidate but its clinical assessment has been limited up to recently due to technological limitations related to the systematic extraction and reconstruction of the fetal signal from the abdominal mixture. With the recent advances statistical signal analysis and machine learning, new algorithms for reconstructing the signal of interest and assessing fetal antepartum and intrapartum health are being elaborated and show promising avenues for new research and application. In particular, we have pointed out relevant heart rate variability measures such as the scattering transform, the decelerative capacity of the phase rectified signal averaging, approximate and sample entropy.

\section{References}

[1] R. Bukowski et al., "Causes of death among stillbirths," JAMA - J. Am. Med. Assoc., vol. 306, no. 22, p. 2459, 2011.

[2] M. F. Macdorman and S. Kirmeyer, "The challenge of fetal mortality.," NCHS Data Brief, 2009.

[3] J. Lopes-Pereira, A. Costa, D. Ayres-De-Campos, C. Costa-Santos, J. Amaral, and J. Bernardes, "Computerized analysis of cardiotocograms and ST signals is associated with significant reductions in hypoxic-ischemic encephalopathy and cesarean delivery: an observational study in 38,466 deliveries," Am. J. Obstet. Gynecol., vol. 220, no. 3, pp. 269-e1, 2019.

[4] S. J. Parker, M. Kuzniewicz, H. Niki, and Y. W. Wu, "Antenatal and Intrapartum Risk Factors for HypoxicIschemic Encephalopathy in a US Birth Cohort," $J$. Pediatr., vol. 203, pp. 163-169, 2018.

[5] M. Cremer, "Über die Direkte Ableitung der Aktionstrome des Menschlichen Herzens vom Oesophagus und Über das Elektrokardiogramm des Fetus," Münchener Medizinische Wochenschrift, vol. 56, pp. 811-813, 1906.

[6] D. S. Hillis, "Attachment for the stethoscope," J. Am. Med. Assoc., vol. 68, no. 12, p. 910, 2011.

[7] E. H. Hon, "The electronic evaluation of the fetal heart rate: Preliminary report," Am. J. Obstet. Gynecol., vol. 75, no. 6, pp. 1215-1230, 1996. 
[8] L. A. Miller, D. A. Miller, and S. M. Tucker, Mosby's Pocket Guide to Fetal Monitoring: A Multidisciplinary Approach, 7th ed. 2009.

[9] J. V. Schmidt and P. R. McCartney, "History and development of fetal heart assessment: A composite," $J$. Obstet. Gynecol. Neonatal Nurs., vol. 29, no. 3, pp. 295305, 2000.

[10] Z. Alfirevic, D. Devane, G. M. L. Gyte, and A. Cuthbert, "Continuous cardiotocography (CTG) as a form of electronic fetal monitoring (EFM) for fetal assessment during labour," Cochrane Database Syst. Rev., 2017.

[11] D. Ayres-De-Campos, C. Y. Spong, and E. Chandraharan, "FIGO consensus guidelines on intrapartum fetal monitoring: Cardiotocography," Int. J. Gynecol. Obstet., vol. 131, no. 1, pp. 13-24, 2015.

[12] S. L. Bloom et al., "Fetal pulse oximetry and cesarean delivery," N. Engl. J. Med., vol. 355, no. 21, pp. 2195 2202, 2006.

[13] J. P. Neilson, "Fetal electrocardiogram (ECG) for fetal monitoring during labour," Cochrane Database Syst. Rev., vol. 12, 2015.

[14] J. Behar, F. Andreotti, S. Zaunseder, J. Oster, and G. D. Clifford, "A practical guide to non-invasive foetal electrocardiogram extraction and analysis," Physiol. Meas., vol. 37, no. 5, 2016.

[15] E. Keenan, C. K. Karmakar, and M. Palaniswami, "The effects of asymmetric volume conductor modeling on non-invasive fetal ECG extraction," Physiol. Meas., vol. 39, no. 10, p. 105013, 2018.

[16] R. Kahankova et al., "A review of signal processing techniques for non-invasive fetal electrocardiography," Accept. IEEE Rev. Biomed. Eng., 2019.

[17] G. S. Dawes, G. H. A. Visser, J. D. S. Goodman, and C. W. G. Redman, "Numerical analysis of the human fetal heart rate: The quality of ultrasound records," Am. J. Obstet. Gynecol., vol. 141, no. 1, pp. 43-52, 1981.

[18] J. Pardey, M. Moulden, and C. W. Redman, “A computer system for the numerical analysis of nonstress tests," Am. J. Obstet. Gynecol., vol. 186, no. 5, pp. 10951103, 2002.

[19] A. Georgieva, C. W. G. Redman, and A. T. Papageorghiou, "Computerized data-driven interpretation of the intrapartum cardiotocogram: a cohort study," Acta Obstet. Gynecol. Scand., vol. 96, no. 7, pp. 883-891, 2017.

[20] A. Bauer et al., "Phase-rectified signal averaging detects quasi-periodicities in non-stationary data," Phys. A Stat. Mech. its Appl., 2006.

[21] D. Ayres-De-Campos, P. Sousa, A. Costa, and J. Bernardes, "Omniview-SisPorto® 3.5 - A central fetal monitoring station with online alerts based on computerized cardiotocogram $+\mathrm{ST}$ event analysis," $J$. Perinat. Med., vol. 36, no. 3, pp. 260-264, 2008.

[22] C. Elliott, P. A. Warrick, E. Graham, and E. F. Hamilton, "Graded classification of fetal heart rate tracings: association with neonatal metabolic acidosis and neurologic morbidity," Am. J. Obstet. Gynecol., vol. 202, no. 3, pp. 258-e1, 2010.

[23] P. A. Warrick, E. F. Hamilton, D. Precup, and R. E. Kearney, "Identification of the dynamic relationship between intrapartum uterine pressure and fetal heart rate for normal and hypoxic fetuses," IEEE Trans. Biomed. Eng., vol. 56, no. 6, pp. 1587-1597, 2009.

[24] A. Georgieva et al., "Computer-based intrapartum fetal monitoring and beyond: A review of the 2nd Workshop on Signal Processing and Monitoring in Labor (October 2017, Oxford, UK)," Acta Obstet. Gynecol. Scand., 2019.

[25] M. Niknazar, B. Rivet, and C. Jutten, "Fetal ECG extraction by extended state kalman filtering based on single-channel recordings," IEEE Trans. Biomed. Eng., vol. 60, no. 5, pp. 1345-1352, 2013.

[26] J. Behar, F. Andreotti, J. Oster, and G. D. Clifford, "A Bayesian filtering framework for accurate extracting of the non-invasive FECG morphology," in Comp. Card., 2014, vol. 41.

[27] J. Behar et al., "Evaluation of the fetal QT interval using non-invasive fetal ECG technology.," Physiol Meas, vol. 37, no. 9, pp. 1392-403, 2016.

[28] G. D. Clifford, R. Sameni, J. Ward, J. Robinson, and A. J. Wolfberg, "Clinically accurate fetal ECG parameters acquired from maternal abdominal sensors," Am J Obs. Gynecol, vol. 205, no. 1, pp. 47.e1-47.e5, 2011.

[29] I. Silva et al., "Noninvasive fetal ECG: The PhysioNet/Computing in Cardiology Challenge 2013," in Comp. Card., 2013, vol. 40.

[30] G. J. J. Warmerdam, R. Vullings, L. Schmitt, J. O. E. H. Van Laar, and J. W. M. Bergmans, "Hierarchical probabilistic framework for fetal R-peak detection, using ECG waveform and heart rate information," IEEE Trans. Signal Process., 2018.

[31] J. A. Behar, L. Bonnemains, V. Shulgin, J. Oster, O. Ostras, and I. Lakhno, "Noninvasive fetal electrocardiography for the detection of fetal arrhythmias," Prenat. Diagn., vol. 39, no. 3, pp. 178$187,2019$.

[32] V. Chudacek, J. Anden, S. Mallat, P. Abry, and M. Doret, "Scattering transform for intrapartum fetal heart rate variability fractal analysis: A case-control study," IEEE Trans. Biomed. Eng., vol. 61, no. 4, pp. 11001108, 2014.

[33] M. Ferrario, M. G. Signorini, and G. Magenes, "Complexity analysis of the fetal heart rate variability: Early identification of severe intrauterine growthrestricted fetuses," Med. Biol. Eng. Comput., vol. 47, no. 9, pp. 911-919, 2009.

[34] D. Hoyer et al., "Heart rate variability categories of fluctuation amplitude and complexity: diagnostic markers of fetal development and its disturbances," Physiol. Meas., vol. 40, 2019.

Joachim A. Behar, PhD

Technion-IIT, Haifa, Israel

Artificial Intelligence in Medicine Lab.

http://aim-lab.github.io

jbehar@technion.ac.il 